\title{
Dubbing practices in Europe: localisation beats globalisation ${ }^{1}$
}

\author{
Frederic Chaume \\ Universitat Jaume I
}

Whereas new technologies seem to favour globalisation in many areas of translation, dubbing shows a reluctance to embrace this trend of globalisation. Translation memories are now used to make translation easier and faster all over the world. In the area of audiovisual translation, new subtitling software has been developed, which is now widely used among both practitioners and companies. Also in subtitling, most microtextual practices (line segmentation, subtitle segmentation, typographical usages, synthesis of information, etc.) are followed by the majority of professionals. But dubbing seems to refuse to bend to homogenisation. Perhaps due to notions of nationalism and singularity attached to this concept, dubbing still shows different macro- and microtextual practices in the European countries in which it is the most popular type of audiovisual translation.

This paper examines four different dubbing practices at a microtextual level -those carried out in Germany, Italy, Spain and France. Before considering new failed attempts to globalise this practice, and also some major advances brought by new technologies, the paper focuses on the differences in layout, take segmentation and dialogue writing in these four countries. These differences show that dubbing practices are still very conservative, and resistant to change and homogenisation.

\section{Introduction: dubbing vs. subtitling in the global world}

New technologies seem to favour globalisation in many translation domains all over the world. Translation memories are now used to shorten translation processes and avoid repetitive routines in legal, administrative, medical or scientific translation, to mention just a few areas. In the field of audiovisual translation new software has been developed for subtitling and is being used among practitioners and companies, particularly to shorten the spotting process and to make subtitling a less-time consuming and therefore more profitable activity. Free software is also available on the web for use by freelancers, companies or universities, Subtitle Workshop being just one example. Such software is increasingly used by both translators and translator trainees, making translation processes more homogeneous across the globe. Also, beta versions of lucrative software can be downloaded without charge, enabling translator trainees to become acquainted with the programs they will be using when they work for subtitling companies. This eventually results in a more homogeneous and global process, since everybody applies the same routines, files are compatible all over the world, and professionals 
complete the same stages to achieve the final product, thereby making translation more profitable and easier. Moreover, in this specific field of subtitling, most guidelines for microtextual practices (with respect to line segmentation, subtitle segmentation, typographical usages, techniques of information synthesis, etc.) are increasingly being followed by the vast majority of professionals and teachers. This is due to the excellent manuals in the market which claim for good subtitling standards (Ivarsson 1992; Ivarsson and Carroll 1998; Díaz Cintas 2003). Díaz Cintas and Remael (2007) will become a cornerstone in the field of subtitling in this respect.

However, this is not the case in the field of dubbing. Dubbing still shows major different macro - and microtextual practices in the European countries in which it is the most popular type of audiovisual translation, to say nothing of Asian dubbing countries. In this paper, I examine various dubbing practices at a microtextual level, and others at a macrotextual level, followed in France, Germany, Italy, and Spain. I focus on the dubbing process, text segmentation, and dubbing symbols.

\section{The dubbing process}

The dubbing process in Western European dubbing countries has been meticulously explained in Whitman (1992). Whereas Germany, Spain and Italy generally seem to follow the same routines and use the same human agents (Spain being the country where fewer human agents are used in the whole process), France deviates from the norm and constitutes the exception by using a unique form of text synchronisation, known as la bande rythmo. Generally speaking, Western European dubbing practices follow the routine described below.

- A TV channel or a distributor decides to broadcast a foreign audiovisual text (mainly films, TV series, documentaries, cartoons).

- A dubbing studio is charged with the task of dubbing it into the target language.

- The dubbing studio finds a translator and organises the whole production process (dubbing director, dubbing actors, sound engineer, etc.).

- The translator produces a rough translation, although now translators are increasingly writing dialogues, thus making this activity more profitable by speeding up the process and reducing costs.

- The rough translation is domesticated by a dialogue writer to make the dialogues sound natural and synchronise the text to the screen characters' mouths. These two tasks may also be done by different people.

- The dubbing actors read this synchronised, more natural translation aloud, under the supervision of the dubbing director and with the help of the sound engineer. In this process, other people may be involved in single tasks, such as the dubbing assistant in Germany or the assistente 
al doppiaggio (dubbing assistant) in charge of take segmentation and the working plan in Italy.

- The sound engineer records the dialogues and the dubbing studio sends the film back to the client with a new dubbing track in which dialogues (and maybe songs, or special effects) are recorded in the target language.

Dubbing actors read the final version of the translation aloud. This final version is printed and placed on a lectern inside the dubbing cabin. Usually, the dubbing director has one copy, the sound engineer has another copy and the dubbing actors have a third copy on the lectern, so that they are free to make gestures with their head and arms (imitating the screen actors' body movements), and they can also breath freely either standing up or sitting on a stool.

This, however, is not the case in France. Here, the translation is typed onto the screen in a track called la bande rythmo. This track is added onto the film for dubbing purposes only and will never be seen by the final spectator. The track is added at the bottom of the screen - like the old black box in subtitling - and dialogues and dubbing symbols are typed into it, so that dubbing actors can read the dialogue lines aloud while they are looking at the screen and hearing the original actors.

Essentially, dialogue lines run from right to left, like scrolling and paint-on subtitles. When the text crosses a red (green, black) vertical line in the middle of the track (barre de précision) it signals to the actors that they should begin reading. The words are meticulously synchronised, so the actors have to wait until these words cross the red line to begin speaking. If there is a pause or a silence between utterances, no dialogue lines will cross the red line until the pause is finished. In addition, the détecteurs (people who are responsible for synchronising the dialogues) may also add dubbing symbols to prepare actors to utter a bilabial in a close-up or to speed up their performance, for example. We examine dubbing symbols further in the fourth section of this paper.

In summary, although some minor differences in the dubbing process are noted across Europe - especially in the number of human agents involved - the most significant difference is the French bande rythmo, and the subsequent time and money invested in trying to improve the dubbing process. Professionals in Spain, Germany or Italy are somewhat critical of this way of working, since they claim it is too expensive and timeconsuming because a person is required to re-type the translation and add dubbing symbols in this new track. They also claim that the process does not necessarily improve the final product, since dubbing actors could read both the bande rythmo and a printed version of a translation placed on a lectern without any discernable difference, as the acting does not depend on where the dialogue lines are located. However, no one appears to be willing to abandon their trusted practice. In the French case, as technical advances 
are being developed and applied to the bande rythmo, we cannot envisage convergence and the dubbing process will remain localised for many years.

\section{Text segmentation}

Another important difference is text segmentation. If we agree that a subtitle is the subtitling unit, then a take is the dubbing unit. In dubbing, the translation is divided into segments, i.e. chunks of text called takes. The takes, also called anelli in Italy or boucles in France, are portions of text with different lengths and layouts in Western European dubbing countries. Historically, translations were divided into short portions of text because dubbing actors had to memorise all the dialogue lines they were to utter. Dialogues were split into these dubbing units, known as takes, enabling dubbing actors to concentrate fully on the screen actors' movements and mouths. If their lines were too long, actors would have to look down at the printed version of the translation in order to read their lines aloud. This would result in their missing the screen actors' mouth articulation and kinesic movements and, eventually, in blatant dischronies regarding isochrony or equal duration of utterances. With shorter dialogue lines, actors could memorise short portions of text and therefore give their full attention to the original actors' body movements and mouth articulation. This eventually results in better synchronisation and a faster pace in dubbing sessions. The faster dialogues can be dubbed and recorded, the lower the dubbing costs are.

For this reason takes have a limited number of lines. However, this number varies from country to country. Again, globalisation has not spread to dubbing practices.

Spain

In Spain the target text is divided every ten lines or eight lines in some Catalonian dubbing studios. This operation involves certain constraints; if this were not the case, a computer could carry out the task at no cost. For instance, a take can be ten lines long, but no one character in the take should speak for more than five lines. That is to say, a take can have ten lines when no dubbing actor has more than five lines to dub. Ten-line takes only occur in the case of two characters speaking five lines each, or three characters speaking three, four and three lines, respectively, and so forth. At the same time, if there is a silence or pause in the film where no dialogues or paralinguistic gestures are heard, the dialogue writer will cut the take and begin a new one after the silence.

Finally, the audiovisual narrative must also be taken into account. What in Film Theory are known as audiovisual punctuation marks, i.e., wipes, iris, fade-ins, fade-outs, etc., also oblige the dialogue writer to segment the text, since a new scene or sequence will begin after them. This will theoretically imply a new mood for dubbing actors, or the introduction 
of new dubbing actors for the new characters on screen. An audiovisual punctuation mark, then, generates a new take. Scene cuts and sequence cuts marked by these audiovisual devices represent the end of one take and the beginning of another. Temporal, spatial and narrative cuts in the plot also require a new take (Chaume, 2004a).

Actors are paid according to the number of takes they dub, according to the number of lines they read aloud, or according to the number of sessions they attend. In Spain, for instance, actors and dubbing directors are paid according to the number of takes they dub; therefore, the more takes a translated film has, the more they earn. Thus, dubbing studios prefer longer takes, since this means that they pay less for the dubbing. In short, whereas dubbing actors and directors prefer short takes because they are easy to remember and record, on the one hand, and because they earn more, dubbing managers prefer longer takes because their costs are lower for the same film.

Hence, while all the above-mentioned constraints (total number of lines, lines per character, silences, scene and sequence cuts, etc.) have to be taken into account, over-segmenting the text into too many takes must also be avoided, since managers prefer longer takes and cheaper dubbings. Consequently, a balance must be reached between the number of takes and the requirements of the audiovisual narrative. Translators and dialogue writers have to consider all these points in order to submit a product that complies with both professional constraints and audiovisual narration.

The following example illustrates some of these conventions:

07.46

YURTXENKO (ON) Hola a tots, hola Steve, mira estos són els meus companys. / Assenteu-vos.

STEVE (OFF) Ens aniria molt bé que ens digueres què saps sobre les rutes de comerç il.legal en esta zona.

YURTXENKO (ON) Estem en Slavyanka. El tràfic d'animals va cap a Usserisk o cap a la frontera de la Xina.

STEVE (OFF) D'ací van directament a la Xina, hi ha una carretera que hi va directa.

\subsection{8 .19}

YURTXENKO (ON) Sí, hi ha una carretera, i també una via de tren. Potser que primer els productes del tigre o del lleopard vagen a Usserisk, i que després, a través d'ací, vagen a la frontera.

STEVE (OFF) Ens centrem en les zones més riques des del punt de vista de la biodiversitat, zones amb poca regulacio jurídica, on els caçadors saben que el risc és menor i els guanys majors. $^{2}$ 
In the example, the first take has eight lines and no one character speaks more than five lines. The second take also has eight lines, and the characters involved have four lines each. There is no audiovisual punctuation mark that obliges to generate a new take, since the camera focuses Yurtxenko for the whole scene.

In Spain, some dubbing studios have developed non-commercial software to segment the translation automatically. This software usually counts the total number of lines and characters in the target text, and then divides the translation according to the maximum number of dialogue lines allowed per take. This process disregards both the requirements of audiovisual narration and the film plot. It is merely an easy way to segment the text automatically, with no consideration for how the action progresses or how scenes and sequences structure the plot and the way they have been joined together. Moreover, this software is not available in the market: dubbing studios hire a computer science engineer who designs the program. The software is only used by the dubbing studio that initially commissioned and paid for it. Again, this is just further evidence to show that dubbing practices are not globalised, but trapped, isolated and hidden away jealously within each dubbing studio.

\section{France}

In France, takes or boucles can be much longer, but they are usually less than a minute long. There is no established limit for the number of lines permitted per take. Instead, dialogue writers meticulously follow audiovisual narrative criteria: action breaks, scene changes, or fade-ins and outs, segment the target text, and a new take begins after these audiovisual punctuation marks. The French tradition also goes further. It also differs in that they consider the following actions to be criteria for introducing new takes as well: a new character comes into the scene (for example, two characters speaking in a scene are joined by a third character), a character leaves the scene or there is a flash back or a flash forward.

Like subtitles, takes usually have a time code in and a time code out. The time in is noted when the first character in the take speaks for the first time, i.e., begins his or her dialogue lines or utters a paralinguistic sign (coughs, laughs, etc.). When the last character in the take stops speaking or uttering suprasegmental features, the time out is noted. The total time duration is also noted. The following example shows a French boucle. 
Boucle: 1 de 10:00:00:00 à 10:00:58:22 Durée: 00:00:58:22

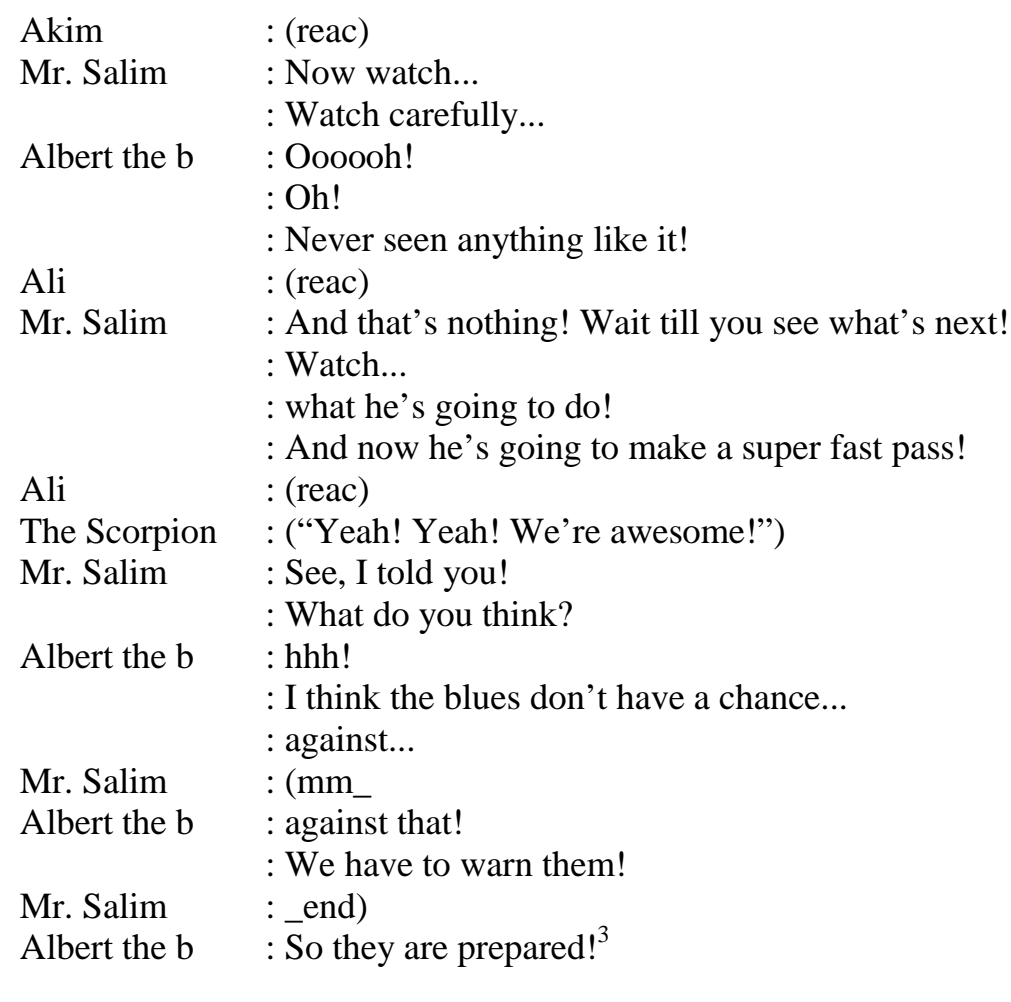

\section{Germany}

As in the previous cases, it is difficult to generalise too much since conventions can vary from one dubbing studio to another. However, general trends can be observed in how the target text is segmented. As in Spain, there is a maximum number of lines per take. German practice does not normally allow takes with more than five dialogue lines. Regardless of the number of lines, the translation is also segmented according to time. Thus, takes can be from 3 to 10 seconds long, and the text is segmented again after a maximum of 10 seconds.

The German tradition also respects the audiovisual narrative criteria in as far as possible. Therefore, action breaks, scene changes, flashbacks, fades and audiovisual punctuation marks entail a new segmentation of the translation, a new take.

As with the French tradition, a take ends when a new character comes into the scene, or when a character leaves.

The following example shows the layout of a German take. 


$\begin{array}{ll}\text { KOVAC: } & \text { (OFF/ON) Sam! } \\ \text { SAM: } & \text { (OFF/ON) Wir fahren noch mal zu Shelley's, (.) zur Union } \\ & \text { Station (.), zum Park... } \\ \text { KOVAC: } & \text { (über.) Sam! (..) Sam, der Wagen steht... }\end{array}$

This short review shows that dubbing practices are far from unified or homogenised in dubbing countries at this time. On the contrary, it seems that the differences between text segmentation and layout are progressively becoming more pronounced. The number of dialogue lines per take varies from one country to another and even from one dubbing studio to another. The use of time codes is different in each country. The length of the lines is different in each country. Time constraints (duration of takes) are different in each country. In a word, globalisation has not yet reached the dubbing industry.

\section{Dubbing symbols}

Another example of heterogeneity surfaces when one considers dubbing symbols. Dubbing symbols are indications that dialogue writers or dubbing assistants include in the translation to help dubbing actors imitate the screen actors' gestures, paralinguistic signs, pitch, volume, primary qualities of the voice, etc. The most important ones refer to where the voice comes from: $(\mathrm{ON}),(\mathrm{O} / \mathrm{S})$, etc., mean that the actor is speaking on-screen, i.e., that the audience can see the actor's face or body, whereas (OFF), (V/O), etc., indicate that the actor is not in field, that the audience cannot see the actor speaking on screen. Other symbols provide more clues to help the dubbing actor.

\section{Spain}

As with layout, dubbing symbols may even depend on the conventions and preferences of individual dubbing studios. A few commonly used symbols are listed below:

- (OFF) Voice Off, voice uttered out of camera

- (ON) Voice uttered On Screen, in field

- (DE) The character has his/her back to the camera

- (DL) The character is far from the camera

- (SB) The character is on screen, but we cannot see his/her mouth

- (G) Paralinguistic gestures

- (A la vez) Two or more characters speak at a time

- (P) (T) (X) A character interrupts another character 
- (Ambiente) A general shot (schoolyard, hotel hall, etc.) with no dialogues in the original script, but original voices have to be dubbed to create possible coherent dialogues, predictable social interactions etc.

- (R) Laughter

- (LL) The character cries or weeps

- (ATT) The voice comes from a telephone

- (ATR) The voice comes from a radio

- (ATV/ATTv) The voice comes from a TV set

- / Silence or pause in the original dialogues

- // Long silence or pause in the original dialogues

These symbols, among others, are inserted in between the dialogue lines, so that dubbing actors know when they must be uttered - like laughter or crying- or how these dialogue lines have to be uttered - paying attention to lipsync in ON voices, interrupting another character, pausing between two sentences, etc.

\section{France}

As in Spain, different dubbing symbols are used in different dubbing studios. They are inserted in the bande rythmo, and they also help dubbing actors act.

- ON / OFF are only marked on the bande rythmo

- (réac) Gestures (especially those made with a closed mouth)

- Gestures (especially those uttered with an open mouth) can also be transcribed: hum, mm, hhh, ahh...

- (rire) Laughter

- (sanglot) Sobbing

- (ambiance) A general shot (schoolyard, hotel hall, etc.) with no dialogues in the original script, but original voices have to be dubbed to create possible coherent dialogues, predictable social interactions etc.

In addition, some studios use particular dubbing symbols to call the actor's attention to some important dubbing constraints. Thus, bilabials can be marked on the bande rythmo with a symbol placed below the consonant, so that the dubbing actor does not miss the closing of the original actor's lips. For example, in a French dubbing studio the symbol of a bone is placed below those bilabials that are uttered in a close-up, so that the dubbing actor pays attention to make a good lip-sync at that time.

Italy

Fortunately, in Italy symbols are standardised as they all appear in the collective agreement signed by the dubbing actors' association and the dubbing companies. This goes a long way to facilitating the dubbing actors' familiarity with dubbing symbols:

- IC On Screen (in campo);

- $\quad$ FC Off Screen (fuori campo); 
- inIC, finIC Beginning and end of voice on screen, respectively;

- inFC, finFC Beginning and end of voice off screen, respectively;

- SOVR A voice is heard over another voice;

- I Pause between two sentences;

- I/ Pause between two scenes;

- $\quad$ EFF A voice effect (through a TV set, etc.);

- VOCE Inner monologues, the voice of thoughts, dreams, etc.;

- Ant. The dubbing actor has to begin speaking shortly before the original actor begins his or her lines;

- DS The character has his/her back to the camera ;

- Orig. Advises actors and engineers not to dub the original voices, special effects, etc.;

- VERSO Gestures (coughing, kissing, crying, whistling...);

- FIATO Gestures (sighing, huffing, puffing);

- RIDE Laughter;

- RIS Smiling, giggling, etc.

Apart from the fact that dubbing symbols are different because they are abbreviations or words in the target language, which seems reasonable from the point of view of the industry, the most worrying issue is that not all countries use the same symbols for the same occurrences, and that some symbols are used differently in each country. Again, unification of symbols in dubbing countries is difficult to foresee.

The above examples show the difficulties of homogenising the process of translation for dubbing. In subtitling, there are also some minor differences between subtitling companies and countries, but these differences do not prevent professional translators from working for foreign companies. The process of adaptation to foreign companies' conventions is easier, since the differences between subtitling practices are less significant. However, in the field of dubbing these differences are so marked that dubbing studios cannot easily take on work from outside their own countries.

A German subtitling company located in Berlin can translate from English into Italian, or an Italian subtitling company based in Rome can translate from English into Spanish. In contrast, it is not usual to translate from English into Italian outside Italy for dubbing purposes. Indeed, when a German dubbing studio receives a translation brief consisting of, for example, translating a film from English into Italian, they look for an Italian partner in Italy to do the dubbing, and vice-versa.

Indeed, in the field of dubbing, historically, the struggle between globalisation and localisation has taken a different path. Just after talking movies came onto scene, creating the need for translation, the first attempts to solve this problem were not at all localised. Multilingual movies, films in the late twenties and early thirties in which a scene was shot in one language, and then, taking advantage of the same sets (and sometimes actors, and even directors), the same scene was shot in a different language, were all produced in the United States (Izard 1992). However, the beginnings of 
a process of localisation were noted when all European language versions of these movies were moved to Paris, Joinville. A second localising step placed all dubbings in their respective target language countries, creating some local dubbing conventions such as those outlined above. This process seems to contradict the general trend towards globalisation that can be observed in other translation domains, and is particularly significant when compared to the subtitling globalisation process; for example, the increasing use of templates in subtitling all over the world allows translators to work for any subtitling company in the world from home.

\section{Attempts at globalisation}

Nevertheless, in the age of globalisation, some attempts have been made to globalise the process of dubbing. In the acclaimed Languages and the Media conference, which takes place in Berlin every two years, major technological advances on audiovisual translation are regularly presented. In its $5^{\text {th }}$ edition in 2004, brand new software was presented by Elio Zarmati, CEO, VoxWorks Technologies, Inc. A voice conversion technology called ReelVoice was designed by the company to morph any voice into another voice. The basic idea of the software is to imitate the voice of original actors on screen. Thus, dialogues in the target language are recorded, and, later on, the pitch of the voice is manipulated by this software to resemble the original screen actor's voice. The voice of the dubbing actor is morphed to sound exactly like the original actor while retaining all the characteristics of the dubbing actor's performance. The aim of the program is to convey a voice similar to that of the original actors to the target audience; that is, since dubbing prevents the target audience from enjoying the real screen actors' voices, this software is able to imitate the original actor's voice, so that the target audience does not completely miss out on the original voices, which for the original audience are inescapably linked to the original characters.

Although it must be recognised that the demo shown was exceptional in its replication of the original actor's voice, the experiment in itself turned out to be a bit of a let-down. The idea of replicating the original voices focused all the attention on the voices. Equivalence was set according to voices. But the effect of imitating the original voices made the target product sound artificial, strange, and foreign. Dubbing is in itself a domesticating kind of translation, especially compared to subtitling. In the demo, the imitation of voices, pitches and tones, which did not comply with the dubbing tradition in the target language (in this case, Spanish), took the audience outside the film, reminded them that they were listening to a translation, and made them aware that the product was a palimpsest. The voices sounded unnatural, exaggerated, overacted. The imitation itself resulted in a foreignising device which deviated from the (implicit) norms of acting in the dubbing target culture. 
Fandubs are another technological innovation that have opened up a new route to globalisation. Fandubs are dubbings of audiovisual texts broadcast on the web, made by fans. Like fansubs, fandubs are mostly created for anime, Japanese cartoons that are not yet being broadcast in a certain target culture. Fans watch their favourite cartoons on the web and then produce a dubbed version at home, using free software (see below) and carrying out the whole dubbing process: translating and writing the dialogues, lip-sync (Chaume 2004b), revoicing the dialogues, recording them in a new soundtrack, and finally uploading the new product back on the web. Although Japanese anime is the most popular fandub genre, fandubs of other genres can be found, such as sitcoms or TV series. The software used by fandubbers to separate the sound tracks from the original audiovisual text, and then record the fan's own voice on a new virgin track can be, among others, Virtual Dub or the more professional software, Adobe Premiere. This makes the whole process of fandubbing homogeneous, and the results very similar worldwide. It should be emphasised, however, that fandubs are not a commercial threat to the dubbing industry. The two can coexist without any risk to the traditional dubbing industry. Furthermore, the appearance of a popular fandub on the web may be a signal for the dubbing industry to make a professional dubbing of the same source text and to distribute it in the target culture in which the fandub was originally made. Fandubs, in this sense, can act as a catalyst for professional dubbing.

New York University has also developed an application known as Video Rewrite. Video Rewrite uses existing footage to create a new clip of a person mouthing words that she did not speak in the original footage. The application automatically labels the phonemes in the training data and in the new audio track, and then reorders the mouth images in the training footage to match the phoneme sequence of the new audio track. When particular phonemes are unavailable in the training footage, Video Rewrite selects the closest approximations. More information can be obtained from the Video Rewrite webpage.

In the field of teaching (Chaume 2003), new software can also help students to be more aware of the dubbing process. As recently as five years ago, students had to read aloud their translation in order to check whether their target text matched the original actors' mouth movements. These days, software can be used to check the lip-syncing process. Windows Movie Maker, for example, free software which is now available to all Windows users, allows the student to cancel the original soundtrack, and to record his or her own translation on a new soundtrack following the screen actors' mouth articulation and movements,. Once finished, students can check whether their recorded translation fits the original actors' mouth movements by playing the clip with their new soundtrack. If the translation fits, the dubbing is satisfactory and he or she can go on to translate and lip-sync the next utterance. If it does not fit, the student can try rephrasing the translation, shortening the sentence, or making it longer, or maybe adding some bilabials to match the closing movements of the original actor's mouth. Al- 
though this cannot be considered a globalising trend in the field of professional dubbing, this kind of software can undeniably homogenise the process of training in academic settings.

Academic proposals of dubbing standards, as far as translation quality is concerned, can also be understood as a globalising trend attempting to make this type of translation homogeneous. As well as Dries' guidelines (1995), I presented an open list of dubbing standards which can be summarised as follows (Chaume 2006: 5-12):

- good lip-sync ('good' depending on the degree of tolerance in each dubbing culture);

- credible spontaneous dialogues (but not too spontaneous, since while the language of dubbing pretends to be spontaneous, it is very normative indeed);

- coherent translation (coherent as a global text, that is, coherent from a linguistic point of view, but also coherent with the visuals);

- equivalence to the source text (equivalence as defined by Toury 1995);

- technical rigour, for example, avoiding noises in the recording, hearing clearly audible and distinct voices, etc.;

- credible acting, i.e., neither overacting, nor underacting.

These standards, understood as ideal objectives, affect the translation itself, the technical recording process, and the dramatisation in the acting, but they do not influence the dialogue writing process, splitting the translation into takes, the layout of the translation, or the use of dubbing symbols.

\section{Some final remarks}

An initial conclusion can be drawn: dubbing countries follow different conventions in how they split the translation into takes, prepare the translation layout, and use dubbing symbols. While translation for subtitling is becoming increasingly homogeneous and globalised in terms of layout and subtitling conventions, translation for dubbing is still heterogeneous and localised in these terms.

This raises the following question: does dubbing refuse to bend before globalisation? Is it a question of conscious resistance? In my view, it is not a question of resistance to globalisation. The dubbing industry has historically been limited to a small number of dubbing studios that have focused their attention on the art of dubbing, rather than on technological advances. Dubbing studios have concentrated their efforts on acting, direction of actors, and lip-sync. For many years, a good dubbing has been regarded as one in which the acting was neither monotonous nor too exaggerated. Unfortunately, translation has not been given a starring role in this process.

On the other hand, the dubbing industry is a very conservative sector, and reluctant to change. One example is the software developed by each individual Spanish dubbing studio to segment the translation into takes 
automatically. Or the software used in France to type the translation and dubbing symbols on the bande rythmo. This software is not available on the market; it is commissioned by a single studio and only used there. Moreover, translators do not have their own copy of the software; as a rule, they simply submit the translation and someone in the dubbing studio uses the software to segment it. Dubbing companies are afraid of industrial espionage; they fear that other companies may take advantage of their findings.

Finally, little research on dubbing technology is being carried out in universities. This also obstructs the flow of information among dubbing companies.

Sticking to old practices and being impervious to change can also be understood as a planned agenda to prevent academic intrusion. In my opinion, a fear of academic intrusion is evidenced by the fact that some common sense changes have not been implemented. For example, I firmly believe that the task of dialogue writing can be better done by the translators themselves. Whitman (1992) also claimed that these two tasks are best carried out by just one person. Dialogue writers manipulate the draft translation submitted by translators in order to make the translation sound fresher and more natural, and also to attain lip-sync. However, dialogue writers do not always master the source language. While they are very good professionals in what they do and have an excellent command of the target language, especially regarding its oral registers, they can completely change the meaning of the film for the sake of naturalness and lip-sync. Moreover, in economic terms, the whole process would be much faster and cheaper if both the translation and the dialogue writing were commissioned to just one individual.

On the other hand, translators are trained in universities, whereas dialogue writers have no official training. They learn their trade by watching other professionals. Courses on dialogue writing for dubbing are very scarce. This increases the risk of atomisation, in the sense that each professional and each dubbing studio have their own conventions. By contrast, in universities translators can be trained, with more or less success, not only with respect to translation strategies and techniques, but also in new technological advances. If translators were allowed to do the dialogue writing, they would share their know-how and experience more easily. Indeed, information flows better among translators than among dialogue writers and dubbing companies. Moreover, if dialogue writing is taught in universities this will no doubt change the pace of old localised practices as well. Undoubtedly, dialogues written by translators would help to homogenise and globalise dubbing practices and cast off old local and capricious conventions.

Can nationalism also be blamed for this heterogeneity? As a domesticating practice, dubbing is more sensitive to nationalistic influences than subtitling, for example. Furthermore, each dubbing country is proud of its dubbing industry, claiming that dubbing is done better there than in other dubbing countries; some European dubbing studios despise the perfectionist 
lip-sync practised in the dubbings of the United States, for example. However, this is not a question of nationalism, as dubbing itself is not only a nationalistic practice (Chaume 2004a). This is more a question of conservative behaviour, a fear of industrial espionage, and a firm belief that dubbing is ultimately a question of art and craft, which cannot be improved by anyone other than the professionals already involved in this industry.

\section{Bibliography}

Chaume, Frederic (2003). "Teaching audiovisual translation: Some methodological proposals". Pérez González, L. (ed.) Speaking in Tongues: Language across Contexts and Users. Valencia: Publicacions de la Universitat de Valencia, 271-302.

Chaume, Frederic (2004a). Cine y traducción. Madrid: Cátedra.

Chaume, Frederic (2004b). "Synchronization in dubbing: a translational approach". Orero, Pilar (ed.) Topics in audiovisual translation. Amsterdam: Benjamins, 35-52.

Chaume, Frederic (2006). "Los estándares de calidad y la recepción de la traducción audiovisual". Puentes, 6. Hacia nuevas investigaciones en la mediación intercultural. Atrio: Universidad de Granada, 5-12.

Díaz Cintas, Jorge (2003). Teoría y práctica de la subtitulación. Inglés-Español. Barcelona: Ariel. Díaz Cintas, Jorge and Aline Remael (2007). Audiovisual Translation: Subtitling. Manchester: St. Jerome.

Dries, Josephine (1995). Dubbing and Subtitling Guidelines for Production and Distribution. Düsseldorf: The European Institute for the Media.

Ivarsson, Jan (1992). Subtitling for the Media. A Handbook of an Art. Stockolm: Transedit. Ivarsson, Jan and Mary Carroll (1998). Subtitling. Simrishamn: Transedit.

Izard, Natàlia (1992). La traducció cinematogràfica. Barcelona: Publicacions de la Generalitat de Catalunya.

Whitman, Candace (1992). Through the Dubbing Glass. Frankfurt: Peter Lang.

Zarmati, Elio (2004). "Who you see is who you hear in any and all languages: A glimpse into the future of dubbing". Paper given at the $5^{\text {th }}$ International Conference Languages \& The Media, Berlin, November, 2004

\section{Filmography}

ER - Season 12, Episode 1 (2005)

Canon City

USA

Dir. Christopher Chulack
Street Soccer - Episode 19

Trop d'amour tue l'amour / Hot stuff

Australia / France

Dir. Margaret Parkes \& Bruno Bligoux

1 This research has been granted and conducted as a part of the research project HUM 200765518/FILO of the Spanish Ministerio de Educación y Ciencia.

2 Although the translation shown here is in Catalan, the same conventions apply to Spanish translations.

3 I am very grateful to Cécile Carpentier, from Chinkel, Paris, for granting me access to this wonderful dubbing studio and providing this sample. The excerpt comes from Street Soccer, Episode 19, and was commissioned by Tele Images Kids.

4 I am most indebted to Matthias Münterfering, from Deutsche Synchron, Berlin, for providing this sample. The excerpt comes from ER, Episode Title "Canon City". 